\title{
Regularized phase tracker with isophase scanning strategy for analysis of dynamic interferograms of nonwetting droplets under excitation
}

\author{
Lennert R. van den Doel, Peter T. Nagy, Lucas J. van Vliet, and Paul Neitzel
}

\begin{abstract}
The surface of a nonwetting droplet is separated from a solid surface by a continuous supply of a lubricating gas film within the apparent contact region. Under certain conditions, e.g., application of an external excitation force, the gas film thickness can decrease to a level where intermolecular forces cause the droplet to wet the surface. The thickness of the lubricating film can be measured by interferometry. Externally imposed oscillations change the shape of the film, leading to dynamic interference fringes that are recorded with a high-speed CCD camera. We propose a spatiotemporal analysis of the interference patterns based on the regularized phase-tracker method. This well-known method minimizes a cost function to estimate the absolute phase of a single element in the interferogram. A proper scanning method along all elements of the interferogram is necessary to avoid phase estimation errors that will propagate throughout the entire continuous phase image of interest. The scanning method we propose traces along contours of constant phase in the interferogram and does not require segmentation of the interferogram in dark and bright fringes. Results in the form of dynamic height profiles of droplets under excitation obtained by this method are presented. (C) 2005 Optical Society of America
\end{abstract}

OCIS codes: $100.2000,100.2650$.

\section{Introduction}

Permanent nonwetting is the terminology used to describe the phenomenon by which a liquid droplet is inhibited from contacting a normally wetted surface. ${ }^{1}$ A side view of a nonwetting droplet is shown in Fig. 1(a). The nonwetting phenomenon is accomplished by establishing a thin lubricating film of surrounding gas. The lubricating film, typically of the order of a few micrometers in thickness, prevents short-range molecular forces from causing molecular contact between the liquid and the solid. ${ }^{2}$ The exploitation of thermocapillarity, the variation of the liquid's surface tension with temperature, is one mechanism to establish this lubricating film. It induces motion on the liquid-gas interface from warm to cool regions and drives the surrounding gas into the contact region by

L. R. van den Doel (L.R.vandenDoel@tnw.tudelft.nl) and L. J. van Vliet are with the Quantitative Imaging Group, Faculty of Applied Sciences, Delft University of Technology, Lorentzweg 1, NL-2628 CJ, Delft, The Netherlands. P. T. Nagy and P. Neitzel are with the George W. Woodruff School of Mechanical Engineering, Georgia Institute of Technology, Atlanta, Georgia 30332.

Received 28 July 2004; revised manuscript received 9 December 2004; accepted 17 December 2004.

0003-6935/05/142695-10\$15.00/0

(C) 2005 Optical Society of America the viscous no-slip condition (see Fig. 2). Under static conditions, i.e., the droplet pressed against the plate but not sliding along it, the pressure in the lubricating film creates an axisymmetric dimple on the liquid-gas interface as observed by the typical fringe pattern shown in Figs. 3(a)-3(c) and Figs. 4(a)-4(c). The separation distance between the liquid and the solid surfaces measures $\sim 13$ and $\sim 5 \mu \mathrm{m}$ at the center and periphery of the dimple, respectively, when the droplet is squeezed $\sim 300 \mu \mathrm{m}$ toward the glass beyond the point of apparent initial contact. 3 The reliability of the nonwetting system depends on the continuous resupply of gas to the thin lubricating film. Furthermore, the liquid and solid surfaces must maintain a sufficient separation during any external dynamic loading, which can excite capillary waves on the free surface when such forcing is oscillatory.

The characteristics of the capillary waves depend on the frequency of the external forcing. A pinned droplet has an infinite number of vibrational modes, each characterized by high dynamic responses of the free surface as shown in Figs. 1(b) and 1(c). In the case of a nonwetting droplet, the interaction between the lubricating film flow and the interface further complicates the system dynamics.

The mode of interest in the present case is excited at the first system resonance, characterized by a 


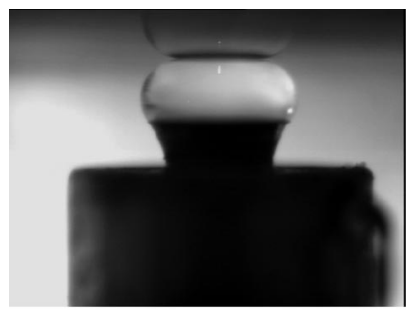

(a)

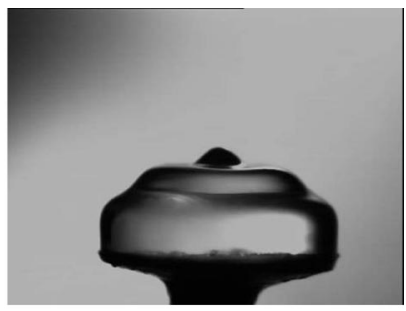

(b)

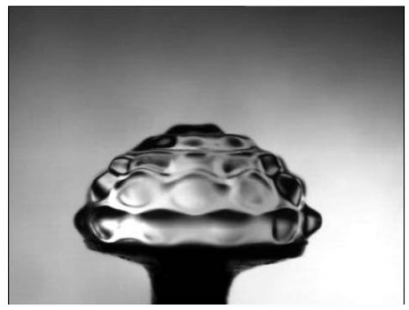

(c)

Fig. 1. (a) Side view of a nonwetting droplet. (b) and (c) Two examples of vibrational modes of a pinned droplet under external dynamic loading.

prolate-oblate oscillation of the interface. Preliminary experiments were carried out to observe the dynamic response of vibrating, isothermal droplets without the presence of an adjacent unwetted plate. A relatively large amplitude response was observed for the first mode oscillation compared with higher modes. One might therefore expect surface oscillations of a nonwetting drop to have large amplitude when excited near this first mode, resulting in an undesirable wetting event. On the other hand, capillary waves on nonwetting droplets damp out quickly because of viscous dissipation. This damping characteristic is another important parameter used to describe the system dynamics. In particular, the nature of this damping influences whether wetting will be observed under given conditions.

To characterize the dynamics of a nonwetting droplet and map out the system response under external forcing, it is desirable to reconstruct the time-varying interface shape within the contact region for specific forcing parameters. Ultimately, this will permit the

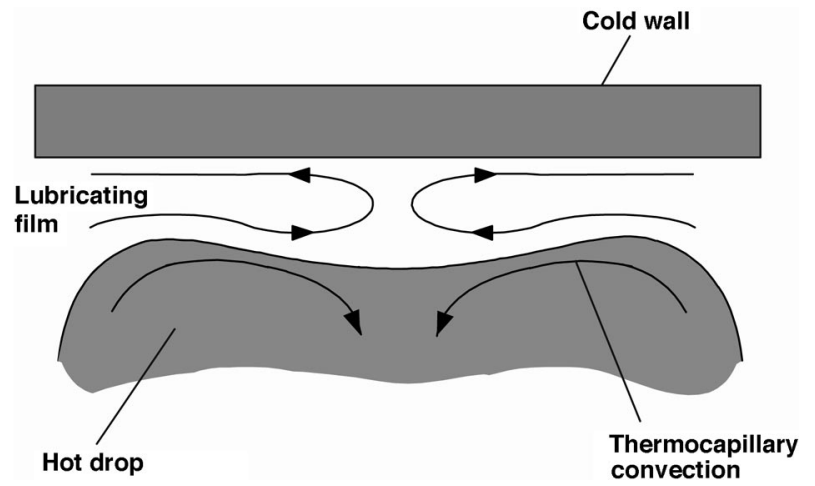

Fig. 2. Exploitation of thermocapillarity establishes a lubricating film that induces motion on the liquid-gas interface and drives the surrounding gas into the contact region.

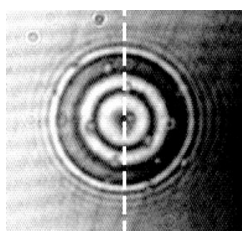

(a)

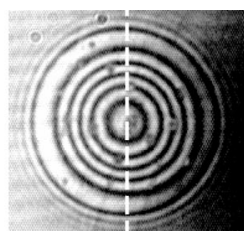

(b)

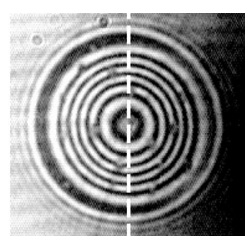

(c)

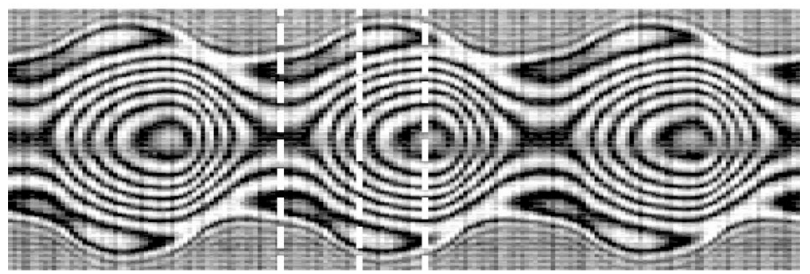

(d)

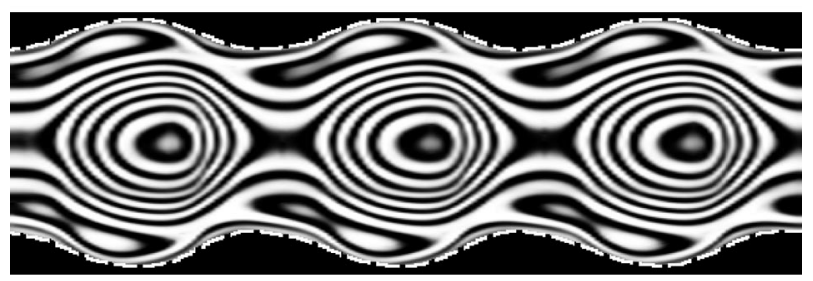

(e)

Fig. 3. (a)-(c) Raw images of dynamic fringe patterns generated by a nonwetting droplet under forced oscillation. Shading, readout noise, and dust particles are clearly present. (d) Space-time representation (time along the horizontal axis) of the image sequence. The dashed lines correspond to the dashed horizontal lines in (a)-(c) through the center of the droplet. The size of the footprint of the droplet changes as a function of time. The closed contours imply that the phase as a function of time increases and decreases, i.e., the droplet increases and decreases. (e) Space-time interferogram after preprocessing. The dashed lines indicate the interferogram's footprint $P$.

determination of the amplification factor, i.e., the ratio of amplitude of the surface to the forcing at a given input frequency, revealing the system response in the spectrum of interest. In addition, this permits the determination of viscously damped behavior following the cessation of external forcing.

\section{Experimental Setup}

The Fizeau interferometer shown in Fig. 5 was used to create fringe patterns within the contact region. A droplet of 10-cs (centistoke) silicone oil (Dow Corning 200 Fluid) was deposited onto a copper disk of $3 \mathrm{~mm}$ in diameter; the disk is machined with a sharp edge and coated with Scotchguard so that the droplet remains pinned to the periphery of the disk. The disk was heated to a temperature $\sim 45^{\circ} \mathrm{C}$, and the heated droplet was squeezed against a cold glass surface maintained at a temperature $\sim 20{ }^{\circ} \mathrm{C}$. As a result of the temperature difference between the droplet and the glass surface, a state of permanent thermocapillary-driven nonwetting was successfully achieved. A 5-mW $\mathrm{He}-\mathrm{Ne}$ laser (Melles-Griot 25LHR151) with a wavelength of $632.8 \mathrm{~nm}$ was passed through a diffusing medium to illuminate the 


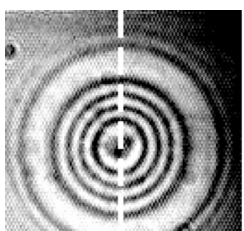

(a)

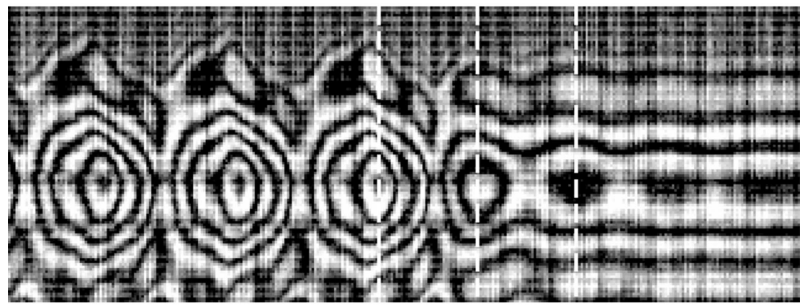

(d)

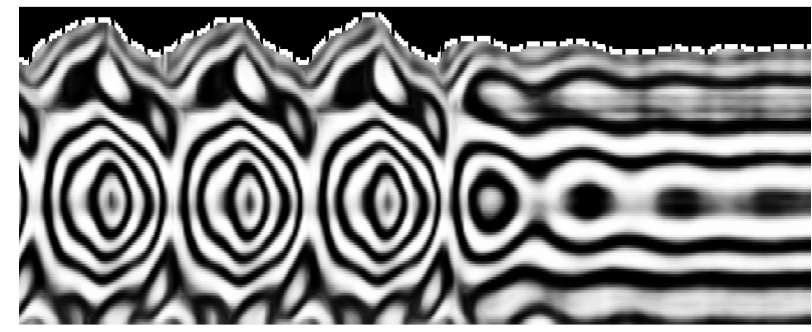

(e)

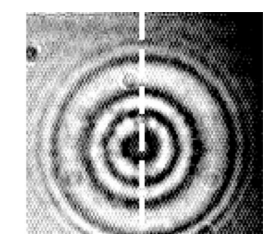

(c) (b)
Fig. 4. As in Fig. 3, but here the amplitude of the oscillation is damped.

contact region. A magnification lens (Navitar Zoom $6000)$ and $2 \times$ extension adaptor from D.O. Industries (catalog number 6030) were attached in front of the high-speed CCD (Kodak Motion Corder Analyzer SRUltra), capable of recording data at 10,000 frames/s. The external forcing was introduced by a loudspeaker driven by a function generator, providing a sinusoidal forcing of the speaker membrane that is transmitted through the droplet support to its surface. Two separate dynamic interference patterns were captured,

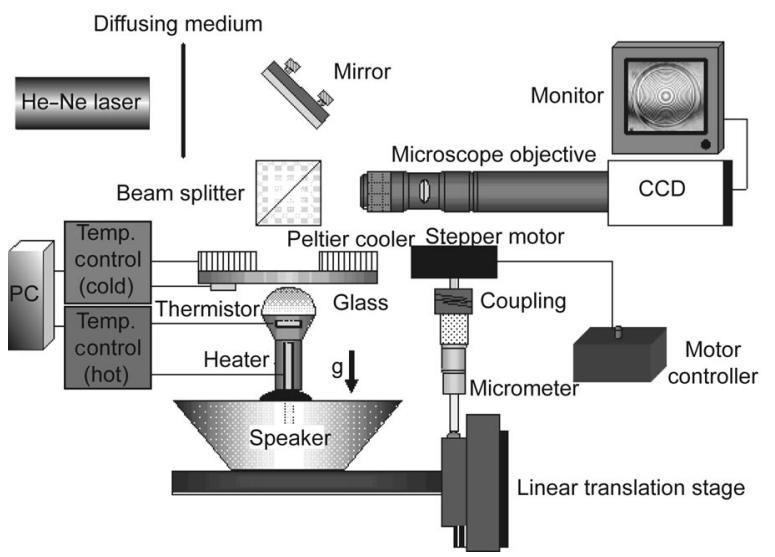

Fig. 5. Experimental setup. A dynamic interference pattern, generated at the surface of the droplet, is captured with a high-speed CCD camera. Vibrational modes of the droplet are induced by a function generator-driven speaker. one during continuous forcing (referred to as forced oscillation) and another right after the forcing was shut off (referred to as damped oscillation). The droplets were excited at $\sim 17$ and $\sim 54 \mathrm{~Hz}$ to study forced and damped oscillations, respectively, and the images were captured at 1000 and 3000 frames/s, respectively.

\section{Analysis of Dynamic Interferograms}

Three fringe patterns from an extended sequence are shown in Figs. 3(a)-3(c) and in Figs. 4(a)-4(c). Each fringe is an isoheight contour. Figure 3 corresponds to forced oscillation and Fig. 4 to damped oscillation. Figures $3(\mathrm{~d})$ and $4(\mathrm{~d})$ show cross sections through the three-dimensional space-time interferogram. These images show the interference patterns along a line through the center of the droplet as a function of time. Because of rotational symmetry, these interferograms represent the complete dynamic shape of the height profile of the liquid droplets $h(r, t)$ as a function of the radial position $r$ and the time $t$. We analyze these interferograms to find these dynamic height profiles.

Recently we studied dynamic interference patterns of a small evaporating liquid volume. ${ }^{4}$ In that study the liquid volume was confined to a micromachined well with an area of $300 \mu \mathrm{m} \times 300 \mu \mathrm{m}$ and a depth of $6 \mu \mathrm{m}$. As a consequence, the dynamic interference pattern had a fixed width along the spatial coordinate of Figs. 3(d) and 4(d). The outer bright fringes in Figs. 3(d) and 4(d) show that the footprint of the liquid droplet varies continuously. Our temporal phaseunwrapping algorithm ${ }^{4}$ requires, however, a constant reference. This holds only in the center part of the interferogram. Furthermore, the algorithm requires that the interference patterns be sampled sufficiently dense in time to estimate the phase.

A second major difference between these two studies is the monotonicity of the phase along the time dimension: In the case of an evaporating liquid sample, ${ }^{4}$ the height $h(r, t)$ of the air-liquid interface decreases monotonically (even with a constant velocity, $\partial h(r, t) / \partial t=$ constant!), implying a monotonically decreasing absolute phase. In the case of an oscillating liquid droplet, the height of the air-liquid interface goes up and down (with a constant period in the case of a forced oscillation), implying an absolute phase that increases and decreases. This makes phase unwrapping an even more challenging problem, as we depict in Fig. 6. The top part of Fig. 6 shows a typical underdamped oscillation analog to a spring-mass system with an additional damping force proportional to, but in the opposite direction of, the velocity of the mass. Let us assume that this oscillation is in the vertical direction and that fringes of equal height are being generated. The intensity of the fringes is shown in the background of Fig. 6. The recorded signal is shown in the bottom part of Fig. 6 . This fringe pattern follows by sampling the curve in the top part at equidistant intervals along the horizontal axis and placing the corresponding intensities along the vertical axis in the bottom part. Detection of the extrema of 

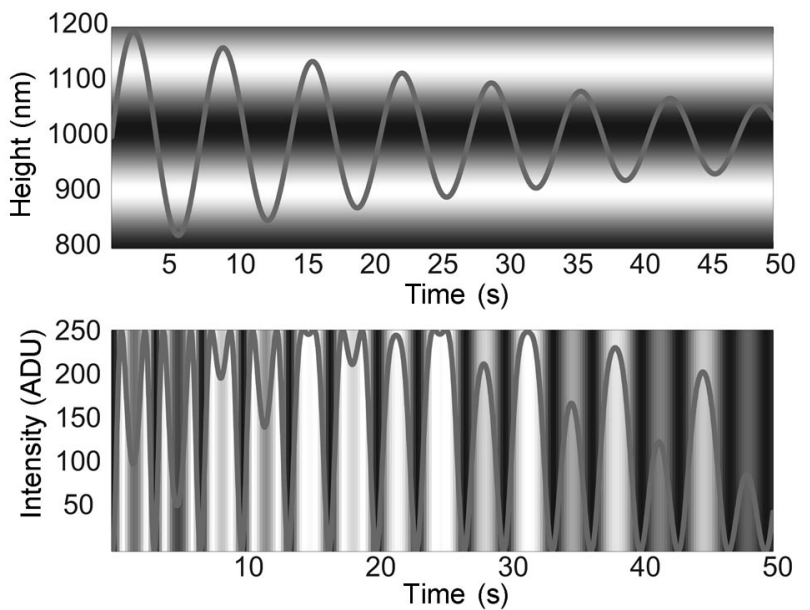

Fig. 6. Top part shows a damped oscillation in the vertical direction as a function of time. The background shows the intensity of the fringes being generated by this damped oscillation. The bottom part shows the recorded interferogram. This interferogram follows by sampling the curve in the top part at equidistant instants and taking the corresponding intensity of the fringes. ADU, analog-todigital unit.

the oscillation in Fig. 6, resulting in a sign change of the phase derivative, is a tedious task for a phaseunwrapping algorithm. This becomes even more complicated when the amplitude of the oscillation extends over multiple fringes.

For these two reasons, we combined the spatial and temporal information into a new interferogram, and we propose to analyze the fringe patterns in Figs. 3(e) and 4(e), which are preprocessed versions of the interferograms shown in Figs. 3(d) and 4(d). We use the regularized phase tracker introduced by Servin et $a l .5,6$ with an isophase scanning strategy.

\section{Preprocessing of Acquired Image Sequences}

Although we record three-dimensional (3-D) spatiotemporal interferograms, we restrict ourselves to the analysis of a two-dimensional cross section, where one dimension is chosen along a spatial coordinate and the second dimension is chosen along the time coordinate. This is done for two reasons. First, the droplet generating the fringe pattern is rotation invariant. A complete $3-\mathrm{D}$ analysis may result in a better signal-to-noise ratio (SNR) because of angular averaging, but not in more information. Second, a 3-D analysis is far more time-consuming because there are approximately 100 times as many points to be processed. Note also that the analysis of a single point in a 3-D interferogram will require a minimization procedure with respect to four parameters, namely, a phase $\left(\phi_{0}\right)$ and three spatial frequencies $\left(\omega_{x}, \omega_{y}\right.$, and $\left.\omega_{t}\right)$ instead of a minimization procedure with respect to three parameters $\left(\phi_{0}, \omega_{r}, \omega_{t}\right)$ as explained in Section 5. The former is computationally more intensive than the latter.

The acquired image sequences are corrupted by a number of (deterministic) noise sources. This calls for specific preprocessing prior to phase estimation.
1. It is clear to see that shading due to nonuniform illumination is present in the images. The contrast in the right side of the images is poor. The shading is removed from each image in the image sequence by fitting a first-order plane $\left[f(x, y)=b_{0}+b_{1} x+b_{2} y\right]$ to a 10-pixel-wide region along the border of the images with a linear least-squares approach. This intensity plane is subtracted from its corresponding image. This approach does not only reduce the intensity variation within the image, but also compensates for intensity fluctuations from image to image.

2. The glass surface between the detector and the droplet contains a number of dust particles or condensation spots. These objects do not move during the image acquisition process, but they cause phase jumps in the interferograms. These phase jumps become apparent as line patterns along the time dimension in the spatiotemporal representation of the 3-D interferogram.

3. The images are corrupted by amplifier and readout noise. The SNR of the 3-D interferogram can be improved by smoothing the interferogram with an adaptive Gaussian filter. ${ }^{7}$ A standard isotropic Gaussian filter smoothes the image in all directions equally, thereby smoothing out the fringe pattern as well. An anisotropic Gaussian filter applied along the fringes of the interferogram reduces intensity variations within a fringe without spurious blurring of neighboring fringes. An adaptive filter uses the local structure in the image to steer the filter. The orientations of the fringes in the interferogram are estimated by an eigenvalue analysis of the gradient structure tensor. ${ }^{8}$ This is a generic tool to describe the local structure and the dimensionality of the structure in images. Locally, the fringes in the 3-D interferogram can be regarded as planelike structures. Planelike structures are defined by the normal vector of the surface. For planelike structures, the eigenvalues of the gradient structure tensor are distributed like $\lambda_{1} \geq \lambda_{2} \approx \lambda_{3}$. Smoothing occurs only in the plane spanned by the eigenvectors related to the two smallest eigenvalues. Another example, where we have successfully applied an adaptive Gaussian filter, can be found in Ref. 9 .

4. After the first three steps, the interferogram can be approximated by $I(x, y ; t)=A(x, y ; t) \cos$ $\left[\phi_{0}(x, y ; t)\right]+B(x, y ; t)$. The analysis based on the regularized phase tracker 6 to retrieve the phase $\phi_{0}(r, t)$ from the interferogram $I(r, t)$ requires that it can be approximated by $I^{\prime}(r, t) \approx \cos \left[\phi_{0}(r, t)\right]$. In the final step we have to remove the nearly static background term $B(x, y ; t)$ and scale the nearly static amplitude $A(x, y ; t)$. The background is removed by subtracting a smoothed version obtained by a large Gaussian filter of the interferogram from itself. The amplitude is scaled to one by applying an error function clipping ${ }^{10}$ to the interferogram:

$$
S_{\text {erf clipping }}(g, t, r)=t+\frac{1}{2} r \operatorname{erf}\left[\sqrt{\pi}(g-t) r^{-1}\right],
$$

where $g$ is the input image, $t$ is the threshold level, 


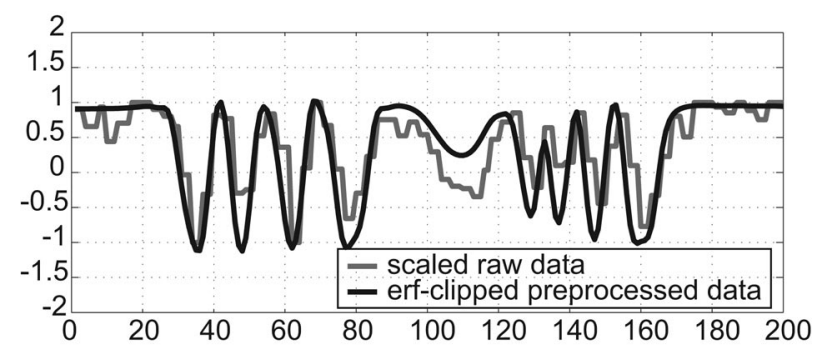

Fig. 7. Contour along the time dimension before preprocessing and after error function (erf) clipping.

and $r$ is the clip range. The dynamic range of the interferogram is approximately between -70 and +60 analog-to-digital units. The pseudothreshold of the error function clip function is set to 0 , and the clip range is set to 50 . Dividing by 25 scales the interferogram to the interval $(-1,1)$. Figure 7 shows a fraction of the interferogram along the time dimension before and after all preprocessing steps. Figures 7, 3(e), and 4(e) show that, after preprocessing, the SNR of the interferogram is significantly improved. This error function clipping avoids sharp transitions in the interferogram. The footprint $P(x, y ; t)$ defining the extent of the interferogram can, for example, be computed by thresholding the interferogram after applying a local variance filter. The local variance of the interferogram is found by first subtracting the local mean from the interferogram, i.e., an isotropic uniform filter with a size approximately equal to the length of one fringe period along the edge of the interferogram. Second, this local difference is squared and finally averaged with the same uniform filter. The resulting image has a strong response where the interferogram is present and a weak response for the background. A global threshold value is chosen halfway between the average local variance of the background and the fringes at the edge of the interferogram. The resulting area of interest $P$ contains small holes that are filled by standard binary image processing operations. Figures 3(e) and 4(e) show the slices of the preprocessed interferograms $I^{\prime}(r, t)$ and indicate the extent of the footprint $P(r, t)$ that we analyze using the algorithm presented in Section 5. All preprocessing steps are computed with DIPimage. ${ }^{11}$

\section{Regularized Phase Tracker with an Isophase Scanning Strategy}

Our analysis of the interferograms $I^{\prime}(r, t)$ shown in Figs. 3(e) and 4(e) is based on the regularized phasetracker algorithm of Servin et al. ${ }^{6}$ The algorithm consists of a phase estimation procedure, which is applied at each element of the interferogram $I^{\prime}(r, t)$, and a scanning strategy that scans over the complete interferogram element by element. Our analysis incorporates exactly the same phase estimation procedure. We propose, however, to use a different scanning procedure. Servin et al. ${ }^{6}$ segment the interferogram into bright and dark fringes by simple thresholding and analyze each fringe object separately, before con- tinuing with the next neighboring fringe object. The complete interferogram is then analyzed fringe by fringe, hence the name fringe-follower regularized phase tracker. The segmentation of the interferogram poses barriers on the scanning of the phases. These barriers can result in large phase jumps along the edges of dark and bright fringes if in the analysis of a fringe object a wrong phase is computed. The scanning strategy that we propose in this paper avoids binarization and scans the interferogram along contours of a constant phase, hence the name isophase scanning.

\section{A. Phase Estimation}

The phase estimation procedure computes the absolute phase $\phi_{0}(r, t)$ at a location $(r, t)$ in the interferogram by minimizing a cost function $U$ over all elements in a rectangular neighborhood $H$ with center point $(r, t)$. For some elements in this neighborhood the phase $\phi_{0}$ is already estimated. These elements are labeled $m(r, t)=1$ and they are taken into account in the phase estimation at $(r, t)$. For the other elements, including the element at $(r, t)$, the phase $\phi_{0}$ is not yet estimated and these elements are labeled $m(r, t)=0$. The cost function $U$ is given by

$$
\begin{aligned}
U(r, t)= & \sum_{(\rho, \tau) \in H}\left\{\left[I^{\prime}(\rho, \tau)-\cos (p)\right]^{2}+\left[I^{\prime}(\rho, \tau)\right.\right. \\
& \left.\left.-\cos \left(p_{1}\right)\right]^{2}+\beta\left[\phi_{0}(\rho, \tau)-p\right]^{2} m(\rho, \tau)\right\},
\end{aligned}
$$

where

$$
\begin{aligned}
p= & p(r, t, \rho, \tau)=\phi_{0}(r, t)+\omega_{r}(r, t)(r-\rho) \\
& +\omega_{t}(r, t)(t-\tau), \\
p_{1}= & p(r, t, \rho, \tau)+\alpha=\left[\phi_{0}(r, t)+\alpha\right] \\
& +\omega_{r}(r, t)(r-\rho)+\omega_{t}(r, t)(t-\tau) .
\end{aligned}
$$

The first term in the cost function $U$ is the difference between the interferogram $I^{\prime}$ and a straight spatialfrequency pattern defined by the first-order plane $p$ with parameters $\phi_{0}, \omega_{r}$, and $\omega_{t}$. This assumes that within the neighborhood $H$, the phase $\phi_{0}$ can be described by a simple plane. This does not only allow for efficient computation of the phase, but also causes some regularization. Obviously, the neighborhood $H$ cannot be taken very large to satisfy this assumption. The second term is the difference between the interferogram and the fringe pattern defined by the plane $p_{1}$, which is parallel to the plane $p$, but shifted by $\alpha$. This second straight spatial-frequency pattern has the same spatial frequencies as the first one, but a slightly different offset. This term is incorporated in the cost function because a slightly phase-shifted interferogram must also look similar to the unshifted interferogram when we are looking for the smoothest phase compatible with the observations. We set $\alpha$ $=0.3 \pi$ in our analyses. The last term in the cost function $U$ is the regularization term, which is the difference between the phase $\phi_{0}$ and the plane $p$. This term is weighted by the regularization parameter $\beta$, and only the elements within the neighborhood $H$ 
where $m=1$, i.e., where the phase is already estimated, are taken into account. This term controls the smoothness and continuity of the detected phase. We set $\beta=0.1$ in our analyses. The cost function $U$ must be minimized with respect to the parameters $\phi_{0}, \omega_{r}$, and $\omega_{t}$. MATLAB's minimization routine (fminunc) requires the derivatives of the cost function $U$ with respect to these parameters. These derivatives follow from Eq. (2) as

$$
\begin{aligned}
\frac{\partial U(r, t)}{\partial \phi_{0}}= & \sum_{(\rho, \tau \in H)}\left\{2\left[I^{\prime}(\rho, \tau)-\cos (p)\right] \sin (p)\right. \\
& +2\left[I^{\prime}(\rho, \tau)-\cos \left(p_{1}\right)\right] \sin \left(p_{1}\right) \\
& \left.-2 \beta\left[\phi_{0}(\rho, \tau)-p\right] m(\rho, \tau)\right\}, \\
\frac{\partial U(r, t)}{\partial \omega_{r}}= & \sum_{(\rho, \tau \in H)}\left\{2\left[I^{\prime}(\rho, \tau)-\cos (p)\right] \rho \sin (p)\right. \\
& +2\left[I^{\prime}(\rho, \tau)-\cos \left(p_{1}\right)\right] \rho \sin \left(p_{1}\right) \\
& \left.-2 \beta\left[\phi_{0}(\rho, \tau)-p\right] m(\rho, \tau)\right\}, \\
\frac{\partial U(r, t)}{\partial \omega_{t}}= & \sum_{(\rho, \tau \in H)}\left\{2\left[I^{\prime}(\rho, \tau)-\cos (p)\right] \tau \sin (p)\right. \\
& +2\left[I^{\prime}(\rho, \tau)-\cos \left(p_{1}\right)\right] \tau \sin \left(p_{1}\right) \\
& \left.-2 \beta\left[\phi_{0}(\rho, \tau)-p\right] \rho m(\rho, \tau)\right\} .
\end{aligned}
$$

The convergence criteria of this minimization routine are the following: The termination tolerance for the input variables $\left(\phi_{0}, \omega_{r}, \omega_{t}\right)$ is $10^{-10}$, and the termination tolerance for the output variable $U$ is $10^{-6}$. The average number of iterations per processed point is approximately six iterations. The minimization of the cost function $U$ amounts to the absolute phase $\phi_{0}(r, t)$ and the spatial-frequency components $\omega_{r}(r, t)$ and $\omega_{t}(r, t)$, all defined at the center point $(r, t)$ of the neighborhood $H$.

\section{B. Isophase Scanning Strategy}

The second part of Servin et al.'s fringe-follower regularized phase tracker ${ }^{6}$ is a scanning strategy along all elements in the interferogram. Basically, the start point acts as a seed point, and the eight neighboring elements of the seed point are analyzed with the parameters $\phi_{0}, \omega_{r}$, and $\omega_{t}$ as start parameters for the minimization of the cost function $U$ of these elements. An undirected scanning strategy, not taking into account any information about the structure of the interferogram, will take the wrong phase branches, and these errors will propagate throughout the entire phase image. Therefore we let our scanning routine follow the fringes in the interferogram, i.e., proceed along the contours of equal phase. We propose a new scanning strategy that does not require segmentation of the interferogram. As a result, our scanning routine requires additional computations, which makes our algorithm more computation intensive. The key idea behind our scanning approach is that the seed point lies somewhere on the phase $\phi_{0}(r, t)$. In the majority of the cases, on one side of the seed point the phase will go down, whereas on the other side the phase will go up. Consequently, we introduce two sets: $R_{+}$for elements in the interferogram with increasing phase with respect to their seed element and $R_{-}$for elements with decreasing phase with respect to their seed element. These sets contain the phase and the coordinates of all candidate seed elements. The candidate seed elements lie on the edge between the analyzed region and the unanalyzed region on the interferogram. Consequently, the maximum size of the sets $R_{+}$and $R_{-}$is of the order of the image width plus the image height, i.e., several hundreds of elements. The elements of each set are sorted in ascending order with respect to the absolute difference with the mean phase of all candidate seed elements in the set. The first element in the sorted set $R_{+}$corresponds to a phase that is the closest to the mean value of all phases in $R_{+}$, and the last element in the sorted set $R_{+}$corresponds to a phase that has the largest deviation from the mean phase. The tracing of the interferogram continues then as follows. The first element in $R_{+}$, the coordinates of which are on the edge of all elements with a currently known phase, is chosen now as a seed point, and only the neighboring elements of this seed point that are not yet analyzed are now analyzed with the phase estimation procedure described above. After each minimization of a single neighboring element, the neighborhood $H$ is updated. This implies that all available information is used in the minimization of the cost function $U$ immediately, rather than putting this new information on hold until all neighboring elements have been analyzed. Again, if the phase difference between the neighboring element and its seed element is positive, then this element is added to $R_{+}$; and if the phase difference is negative, then this element is added to $R_{-}$. If all neighboring elements of the first element in $R_{+}$have been analyzed, then this seed point is removed from $R_{+}$. The next step is to repeat these computations for the first element in the sorted set $R_{-}$. These two steps continue until both sets are empty. Of course it is possible that one of the two sets becomes empty during the tracing of the interferogram. In that case, the analysis continues with only one of the two sets, until the empty set again contains one or more elements. The reason for sorting the sets with respect to the absolute difference with the mean value of all the seed phases is obvious: The seed element to be analyzed will add elements to the sets that are again close to this mean value. The mean phase of the updated set will not deviate much from the previous mean phase, inducing another seed element close to this phase value. This implies that the scanning strategy will follow a constant phase contour (analyzed elements form a closed ringlike area) or trace (analyzed elements do not form a closed ringlike area). Hence the name isophase scanning strategy. Another way of looking at this approach is that the probability that the global phase range will increase is minimal since only new elements are added with a phase around the mean value of the phase. 


\section{Region-of-Support Condition}

Applying this tracing algorithm directly to the interferogram of Fig. 3(e) or 4(e) can result in a thin open isophase contour of analyzed elements. This means that only a few elements will contribute to the minimization of the cost function $U$, especially to the third term of $U$. This can easily introduce errors that will propagate throughout the entire phase $\phi_{0}$. To avoid these errors, we require that at least a fraction $f_{\text {supp }}$ of all elements in the neighborhood $H$ be analyzed before the center point of $H$ is analyzed. We refer to these analyzed elements in $H$ as the region of support. The upper limit for $f_{\text {supp }}$ is $50 \%$, which corresponds to one half of the elements in $H$ that are already analyzed. If the size of $H$ is $11 \times 11$ pixels, then the region-of-support is 60 elements, which is fairly large. A practical lower limit for $f_{\text {supp }}$ is $15 \%-$ $20 \%$, which corresponds to approximately 20 elements for the same size of $H$. The larger the value of $f_{\text {supp }}$, the more stringent this condition is on the scanning routine. This region-of-support condition, however, is not sufficient. The algorithm may get lost, despite the fact that the region-of-support condition is met because of the following reasons: (1) local differences in SNR, (2) the amplitude of the preprocessed interferogram $I^{\prime}$ deviates too much from one, and (3) the interferogram is undersampled (in time). Therefore we impose more conditions on the region of support. The region of support must not only consist of a certain number of elements, but the phases found in the region of support must lie within certain boundaries on a plane.

\section{Higher-Order Phase Models}

The minimization of the cost function $U$ assumes that the phase is locally a plane. A more accurate description of the phase $\phi_{0}$ is a quadratic surface, a thirdorder surface, or a fourth-order surface. This would require, however, minimization of $U$ with respect to 6,10 , or 15 parameters. This does not only slow down the minimization procedure significantly, but the parameter space in which the solution must be found will become even more complex, and it will be hard to find the optimal solution. Furthermore, the variance will increase with a growing number of parameters to be estimated. On the other hand, it is straightforward to fit a second-, a third-, or a fourth-order surface to the phases estimated in the region of support by a least-squares approach. This higher-order surface is fit to the data in the local neighborhood $H$. The main assumption is that the phase map we are looking for is a smooth surface. This implies that the leastsquares fit of the surface must have a small error. The second condition is that the distribution of errors must lie completely in the range $\left(-2 \pi \alpha_{\text {surf }}, 2 \pi \alpha_{\text {surf }}\right)$, where $\alpha_{\text {surf }}$ is a fraction of a fringe period of $2 \pi$. The smaller the value of $\alpha_{\text {surf }}$, the more stringent this condition is on the scanning routine. With a fourthorder surface to describe the phase $\phi_{0}$, we use $\alpha_{\text {surf }}$ $=1 / 45 \approx 0.02$. Furthermore, the fourth-order surface description of the phase map allows for predic- tion of the phase of the element that we are currently analyzing. The third and final demand is that the difference between the predicted phase and the phase found by the minimization of the cost function $U$ is also smaller than $2 \pi \alpha_{\text {surf. }}$. If an element satisfies these three conditions during the analysis, then it will be added to either $R_{+}$or $R_{-}$and it will be labeled as analyzed, $m=1$. Otherwise, it will remain unanalyzed: $m=0$. This implies that some elements can be a candidate seed element for a number of times, until they meet all three conditions. For some elements it will mean that they will never meet the conditions, possibly because of phase estimation errors in their neighborhood. Consequently, not all elements in the interferogram $I^{\prime}$ will be analyzed in the end, depending of the values of $f_{\text {supp }}$ and $\alpha_{\text {surf }}$.

The portion of elements that have not been analyzed at the end of the tracing routine can be analyzed afterwards without considering any of the three conditions as described above. Here only the available neighborhood information is taken into account. The starting parameters can be chosen as the mean values of the analyzed elements in the neighborhood $H$ of these unanalyzed elements. The computed phase map can be further optimized by performing additional minimization scans over the interferogram with the cost function $U$ without the second term, as argued by Servin et al. ${ }^{6}$ Since at this point every element of the interferogram has been analyzed, it is now no longer necessary to follow a specific scanning path through the interferogram. The second and possible subsequent iterations are then identical to those of Servin et al.'s regularized phase tracker. ${ }^{6}$

\section{Experimental Results}

The analyses described in this section were performed on a general-purpose computer equipped with a Pentium 4 processor running at $2400 \mathrm{MHz}$ with 1-Gbyte internal memory. The computations were performed in MATLAB extended with the digital image processing toolbox DIPimage. ${ }^{11}$ The complete preprocessing of the acquired interferograms took of the order of a few minutes. The isophase scanning of the interferogram took of the order of $1 \mathrm{~h}$. The dimensions of the images were as follows: $\approx 150$ elements along the spatial dimension and $\approx 600$ elements along the time dimension for the forced oscillation (Fig. 3) and $\approx 1000$ elements for the damped oscillation (Fig. 4). The number of elements in the area of interest $P$ is around 80,000 and 115,000 elements, respectively.

\section{A. Dynamic Height Profiles of Forced Oscillating Droplets}

We applied our algorithm to the interferogram shown in Fig. 3(e). The seed point was chosen somewhere along one of the closed circular fringes in the center part of the interferogram. The size of the neighborhood $H$ used for the minimization of the cost function $U$ was $11 \times 11$ elements. Furthermore, we required that $f_{\text {supp }}=0.35$ or 40 elements already analyzed. The maximal tolerable error in the prediction with a fourth-order surface was $\alpha_{\text {surf }}=1 / 45$. We accepted the same maximal difference between the predicted 


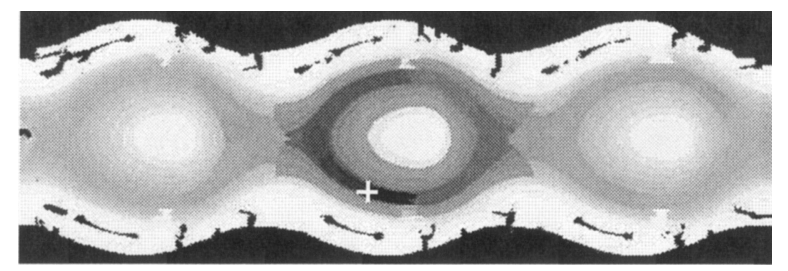

Fig. 8. Illustration of how the scanning is performed. The initial seed point is indicated by a small cross. For visualization purposes only, 17 different scanning regions of unequal size are shown. The scanning is performed from black to white.

phase and the estimated phase based on the minimization of the cost function $U$.

The interferogram shown in Fig. 3(e) has a number of difficult regions, where the algorithm fails. The outer part of the interferogram contains a bright fringe that splits into two fringes that at a later instant connect again and split again and so forth. This implies that the height profile of the liquid-gas interface contains two contours of equal height that come together and separate over and over again. The region in between the two split fringes contains another bright fringe. In the early stage of the splitting the modulation is not high, but in the late stage of the splitting the modulation reaches, coincidentally, the maximum amplitude. In this neighborhood, the regularized phase-tracker algorithm will consider this as a region where the phase monotonically increases or decreases, instead of detecting a maximum or a minimum in the phase. As a consequence, the phase after the first iteration contains a number of regions at the edge where the phase increases and a number of regions where the phase decreases. In between these regions, the algorithm does not meet its requirements and does not estimate the phase at all: $m$ remains zero. Figure 8 illustrates how the scanning is performed during the analysis of the interferogram from Fig. 3(e). The scanning is performed from black to white. For visualization purposes we show only 17 subsequent periods of unequal length. The edges between two subsequent periods lie along isophase contours, hence the name isophase scanning strategy.

We use simple binary image processing steps on the footprint $P$, the mask $m$, and the phase $\phi_{0}$ to mask out the regions with the wrong phases. The regions where the phases are not correctly estimated are manually selected. The presence and locations of these regions depend heavily on the geometry of the interferogram. In this specific case (see Fig. 9), erroneous regions are connected on one side to the edge of the footprint $P$, and on the opposite side they are connected to an isophase contour in the image $\phi_{0}$. On the remaining two sides they are connected to thin indentations, where the phases are not estimated at all, i.e., where $m=0$. The first side of these regions is defined by the footprint $P$, the second side follows from thresholding the phase $\phi_{0}$ at a manually selected isophase value, and other two sides are defined by $m$. In these regions we check for the monotonicity of the phase with respect to the phase of the main

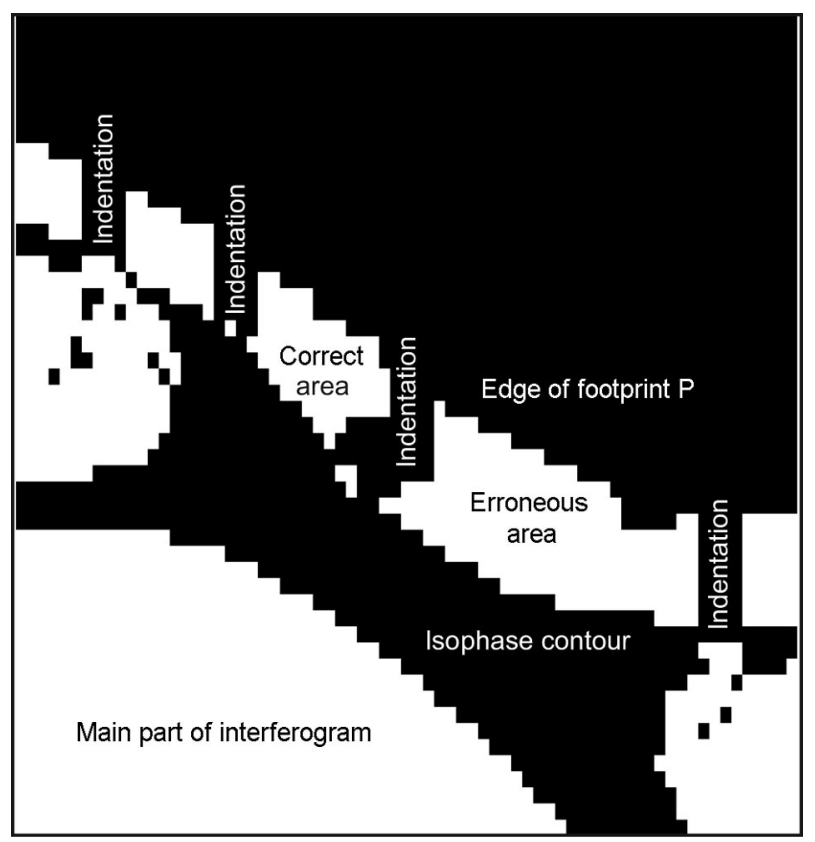

Fig. 9. For this specific case, an erroneous area is bound by the edge of the footprint $P$, a manually selected isophase contour, and two indentations. The erroneous area is removed from the phase.

part of the interferogram (the area on the other side of the isophase contour). To ensure that all the wrong phases are removed, these areas are dilated one or two steps before they are removed from the footprint $P$ and the binary image $m$. The erroneous phases are, of course, also removed from the phase $\phi_{0}$. This defines the modified footprint $P^{\prime}$. In an intermediate iteration, we let the footprint $P^{\prime}$ grow dilation by dilation and update the modified phase accordingly. This resulted in a phase without erroneous regions at the edge. Finally, we applied three iterations of the algorithm with the simple cost function and with a simple line-by-line scanning of the interferogram.

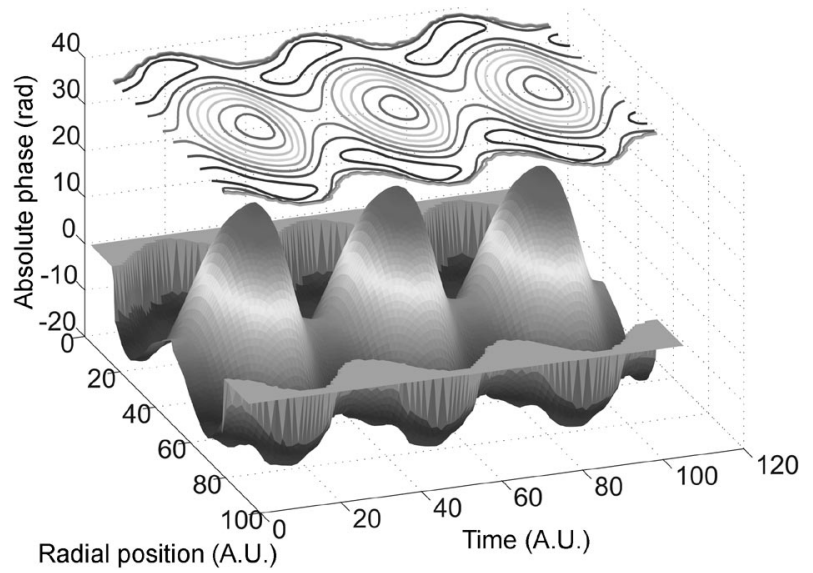

Fig. 10. Final phase $\phi_{0}(r, t)$ of the interferogram shown in Fig. $3(\mathrm{e})$. It can be seen that the footprint of the droplet grows and shrinks. It is difficult to see that the falling of the center point of the droplet is faster than the rising of the center point of the droplet. 


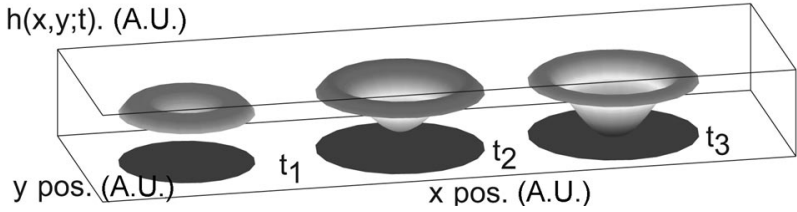

Fig. 11. Three height profiles $h(x, y ; t)$ of the droplet under constant excitation. The instants $t_{1}, t_{2}$, and $t_{3}$ correspond to the interferograms of Figs. 3(a), 3(b), and 3(c), respectively; pos., position.

The final phase $\phi_{0}$ is shown in Fig. 10. The phase $\phi_{0}(r, t)$ can be converted to a relative height map $h(r, t)$ by the relation $\Delta h=\lambda \Delta \Phi / 4 \pi n=\lambda \delta \Phi / 4 \pi$, with $n=1$ the refractive index of air. This formulation relates the changes in height of the droplet's profile to the changes in the phase between two time intervals. The height map is relative since the phase $\phi_{0}=0$ is chosen arbitrarily by the algorithm. There is no reference point to define an absolute phase. The height maps $h(x, y ; t)$ corresponding to the interferograms in Figs. 3(a)-3(c) are shown in Fig. 11.

\section{B. Dynamic Height Profiles of Damped Excited Droplets}

We also analyzed the preprocessed interferogram of Fig. 4(e) with $\alpha_{\text {surf }}=1 / 25$ and $f_{\text {supp }}=0.20$. The top half of the interferogram was flipped and laid over the bottom half to avoid phase estimation errors in the lower edge of Fig. 4(e). Again, the modulation depth of the bright fringe between the two split bright fringes reaches the maximum amplitude and causes

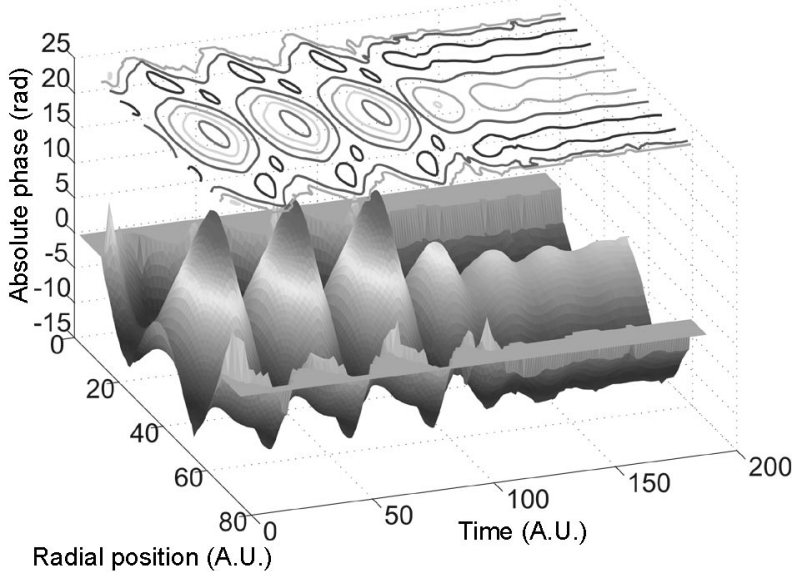

Fig. 12. Final phase $\phi_{0}(r, t)$ of the interferogram shown in Fig. 4(e). It can be seen that the amplitude of the oscillation is fading.

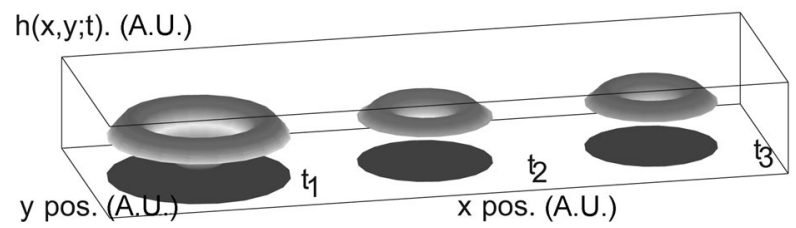

Fig. 13. Three height profiles $h(x, y ; t)$ of the droplet under excitation with fading amplitude. The instants $t_{1}, t_{2}$, and $t_{3}$ correspond to the interferograms of Figs. 4(a), 4(b), and 4(c), respectively; pos., position. the same errors in some regions only as in the case of forced oscillation; the periodicity of the fringe pattern allowed us to replace a part of the phase where the algorithm failed with a good redundant part of the phase. The final result after three additional iterations with the simple cost function and line-by-line scanning is shown in Fig. 12. The small peaks at the sides in Fig. 12 are mainly caused by a bad definition of the footprint $P$. The height maps $h(x, y ; t)$ corresponding to the interferograms in Figs. 4(a)-4(c) are shown in Fig. 13.

\section{Conclusions and Discussion}

In this paper we presented an algorithm to analyze dynamic interference patterns generated by nonwetting droplets under excitation. The surface of a pinned droplet is excited with a function generatordriven speaker. Fringe patterns are generated with near-normal illumination. The experimental setup suffers from shading and dust particles. These effects are successfully removed by application of adaptive digital filtering techniques to the full 3-D interferogram $I(x, y ; t)$. In the current setup, (almost) the complete spatial extent of the interferogram is captured. The algorithm, however, requires only spatial information $I(r, t)$ along a line through the center of the droplet. This allows for complete reconstruction of the height map $h(x, y ; t)$ because of rotation invariance of the droplet shape. The SNR in the step from $I(x, y ; t)$ to $I(r, t)$ can be improved with angular averaging. In this paper we simply took $y=r$ at a constant value of $x$. The limiting factor of our experimental setup is the sampling rate: At the maximum sampling rate of $10,000 \mathrm{frames} / \mathrm{s}$, we must reduce the spatial resolution along one direction and accept a lower SNR of our images. This can, however, still result in an interferogram that contains all the necessary information, the SNR of which can be improved by the same preprocessing steps.

The algorithm we presented in this paper is based on the regularized phase tracker of Servin et al. ${ }^{6} \mathrm{We}$ propose a scanning strategy that scans along contours of constant phase, without the need to segment the interferograms in dark and bright fringes. The performance of the scanning strategy is defined by two parameters: (1) The region of support $f_{\text {supp }}$ defines how many elements in the neighborhood $H$ already have been analyzed before the center element in the neighborhood $H$ can be analyzed (2) and the maximum tolerable error $\alpha_{\text {surf }}$ of a least-squares fit of a fourth-order surface through the phase in the region of support. This error is also used as an upper limit for the error between the predicted phase of the center element in $H$ (based on the least-squares fit) and the estimated phase (based on the minimization of the cost function $U$ ). The algorithm performs well in the regions of the interferograms where the fringes are closed. Some regions are incorrectly analyzed. Manual corrections are required to remove these erroneous regions from the phase. Our scanning strategy is more sensitive to noise compared with the original scanning strategy of Servin et al., ${ }^{6}$ but gain of 
sensitivity is sufficiently compensated for by the preprocessing steps as described in this paper: The input interferogram for the analysis has a high SNR, and after one iteration more than $90 \%$ of the elements have been successfully analyzed. One manual step is required to remove possible erroneous regions before the remaining elements can be analyzed. The strength of our approach is that we analyze only a single (difficult) spatiotemporal interferogram that results directly in the dynamic shape of the droplet's surface $h(r, t)$ instead of analyzing a large series of simple interferograms, which require calibration before the dynamic shape of the droplet's surface $h(x, y ; t)$ can be retrieved.

\section{References}

1. G. P. Neitzel and P. Dell'Aversana, "Noncoalescence and nonwetting behavior of liquids," Annu. Rev. Fluid Mech. 34, 267289 (2002).

2. P. Dell'Aversana, J. R. Banavar, and J. Koplik, "Suppression of coalescence by shear and temperature gradients," Phys. Fluids 8, 15-28 (1996)

3. P. Dell'Aversana, V. Tontodnato, and L. Carotenuto, "Suppression of coalescence and of wetting: the shape of the interstitial film," Phys. Fluids 9, 2475-2485 (1997).

4. L. R. van den Doel and L. J. van Vliet, "Temporal phaseunwrapping algorithm for dynamic interference pattern analysis in interference-contrast microscopy,” Appl. Opt. 40, 4487-4500 (2001)

5. M. Servin, J. L. Marroquin, and F. J. Cuevas, "Demodulation of a single interferogram by use of a two-dimensional regularized phase-tracking technique," Appl. Opt. 36, 4540-4548 (1997).

6. M. Servin, J. L. Marroquin, and F. J. Cuevas, "Fringe-follower regularized phase tracker for demodulation of closed-fringe interferograms," J. Opt. Soc. Am. A 18, 689-695 (2001).

7. W. T. Freeman and E. H. Adelson, "The design and use of steerable filters," IEEE Trans. Pattern Anal. Mach. Intell. 13, 891-906 (1991).

8. G. Granlund and H. Knutsson, Signal Processing for Computer Vision (Kluwer Academic, Dordrecht, The Netherlands, 1995), Chap. 6.

9. B. Rieger, L. R. van den Doel, and L. J. van Vliet, "Ring formation in nanoliter cups: quantitative measurements of flow in micromachined wells," Phys. Rev. E 68, 036312 (2003).

10. L. J. van Vliet and P. W. Verbeek, "Better geometric measurements based on photometric information," In Proceedings of IEEE Instrumentation and Measurement Technology Conference IMTC94 (IEEE, Piscataway, N.J., 1994), pp. 1357-1360.

11. C. L. Luengo Hendriks, L. J. van Vliet, B. Rieger, G. M. P. van Kempen, and M. van Ginkel, "DIPimage: a scientific image processing toolbox for MATLAB" (Quantitative Imaging Group, Faculty of Applied Sciences, Delft University of Technology, Delft, The Netherlands, 1999), http://www.qi.tnw. tudelft.nl/DIPlib. 